\title{
Selective Laser Melting process simulation of open lattice cellular materials
}

\author{
George Lampeas ${ }^{1}$ \\ ${ }^{1}$ Laboratory of Technology and Strength of Materials, Department of Mechanical Engineering and \\ Aeronautics, University of Patras, 26500 Rion, Greece.
}

\begin{abstract}
In the present paper an efficient simulation of an Additive Manufacturing process is presented. The analysis is aiming to quantify the relations between process parameters and material characteristics. The methodology is demonstrated in the fabrication of an open-lattice BodyCentred-Cubic (BCC) cellular core, produced by means of the Additive Manufacturing Selective Laser Melting method. The results of the developed finite element model are compared to experimental results demonstrating a successful validation of the simulation methodology. The results of the present simulation can be used as input for the prediction of the mechanical properties of the cellular materials under investigation.
\end{abstract}

\section{Introduction}

Metallic cellular materials exhibit interesting mechanical properties and may be designed to have reduced weight, as compared to solid materials of same properties. They also exhibit good energy absorption characteristics and good thermal and acoustic insulation properties making them ideal candidate materials for use in the medical, transportation and consumer goods industries. A wide area of application of cellular materials is their use as core materials in sandwich structures, as they offer advantageous behaviour mainly in compression and impact. An interesting category of cellular cores is the open-lattice core, which is typically formed by an interconnected network of solid struts (Ptochos et al. [1]); their main advantage over the conventional honeycomb or foam cores is their ventilation capability, which prevents the absorption of moisture and the subsequent degradation of material properties.

The mechanical properties of lattice structures have been thoroughly investigated. Gumruk et al. [2] used analytical methods in order to evaluate the compressive behaviour of stainless steel BCC micro-lattice structures. The compressive response of a stainless steel core was studied by Labeas et al. [3]; FEM analysis was used in order to calculate the unit cell elasticity modulus, plateau stress and compaction strain. In [1] Ptochos et al. have analytically determined the elasticity modulus, the Poisson's ratio and the shear modulus for a cuboid cellular unit cell with analytical results to be in excellent correlation with the respective numerical ones. Shen et al. [4], have observed that cellular structures containing vertical strands offer superior mechanical performance, during an experimental investigation of the compressive behaviour of lattice structures. In addition to the performance of lattice cellular structures under static loading, their behaviour under 
dynamic loading has been also investigated by several researchers in [5], [6] and [7]. In all the above mentioned studies, the prediction of the lattice cellular structures mechanical behaviour is based on the properties of the base material and the unit cell geometrical and built characteristics.

It is clear that Additive Manufacturing (AM) is advantageous for the production of open-lattice cellular materials and structures over alternative conventional processes, as the later can only build lattice structures of relatively simple geometry, which lack advanced functionalities [8], [9]. Manufacturing of parts by adding material in layers has its roots in the early 1980 s, along with the development of the accompanying technologies, such as computers, lasers and controllers [10], with the first industrial applications taking place in the mid-1980s [11]. AM typically involves layer wise consolidation of material (in powder, liquid or filament form) to create arbitrary geometries. Hence, AM is characterized as solid freeform fabrication, digital manufacturing and e-manufacturing. Key advantages of AM, as opposed to the conventional subtractive technologies for metals processing, have attracted an increased attention by several manufacturing sectors, such as aerospace, marine, automotive, medical and consumer good [12]. The freedom in design, the ability to build parts composed of multiple materials and the almost zero CAD-to-Part time are the main drivers for this transition. Despite the fact that numerous AM process variations have been developed, the most commercialized among them are the Selective Laser Melting (SLM), Electron Beam Melting (EBM) and Selective Laser Sintering (SLS). In SLM, consolidation of powder particles is achieved through full melting of the material. A laser beam is used to provide the heat input required. The effect of processing conditions in additive manufacturing fabricated lattice structures has drawn the attention of researchers, however, still limited research is existing in this field, e.g. [13]. Yan et al. [14] observed that gyroid cellular lattice structures with a wide unit cell size from 2 to $8 \mathrm{~mm}$ can be fabricated by SLM without the need of support structures. The SLM process parameters have to be carefully defined so that the open-lattice geometry, with the selected geometrical characteristics and consequently the required mechanical properties, is produced. To this scope process numerical simulation is very useful, as it results to the reduction of costly and time consuming trial-and-error approach.

The SLM method engages a complex heat and mass transfer process involving conduction, convection and radiation. A number of numerical simulation efforts can be found in the open literature aiming to better understand the basic physics of the SLM related phenomena. Among these works Hussein et al. [15] has analyzed the effect of SLM process parameters, such as layer thickness, preheating temperature, laser power and scan velocity on the melt pool size and the accurate production of solid SLM parts; Foroozmehr et al. [16] performed numerical simulations of a single layer stainless steel 316L SLM process considering a thick powder bed at different scan speeds, concluding that the melt pool dimensions remained constant after the third laser pass; in the work of Hodge et al. [17] the heat source model proposed of Gusarov has been incorporated to develop a complex continuum thermomechanical model for the simulation of SLM process application on $316 \mathrm{~L}$ stainless solid steel parts; this model included a volumetric heat source representing the radiation transport of laser energy into the powder and considerations of phase change of the powder into a consolidated material that takes into account the latent heat of melting and has been used for temperature field melt pool shape predictions. More recently FEM analysis was utilized by Zhuang at al. [18] to simulate the thermal characteristics of TiAl6V4 powder to accurately predict temperature distribution time history and melt pool extent during SLM process. Despite all the efforts for the numerical simulation of SLM process, currently, their is lack of specialized process simulation tools for the specific case of open-lattice cellular materials production. 
In the present work, an efficient computational methodology for open-lattice cellular materials SLM process simulation is presented. The methodology aims to derive relations between process parameters and material characteristics, such that a first-time right process parameters selection is enabled. The simulation method is demonstrated in the SLM fabrication of an open-lattice Body-Centred-Cubic (BCC) unit cell cores. The results of the developed finite element models are successfully compared to experimental results validating the simulation methodology. The simulation output can serve as input to mechanical models in order to predict cellular material mechanical properties.

\section{Description of Cellular Material Additive Manufacturing by SLM}

\subsection{Selective Laser Melting}

The Selective Laser Melting process is based on powder deposition on previous deposited layers (or on the substrate for the first layer) using a rolling mechanism to create the socalled powder bed, as shown in Figure 1. The laser beam selectively scans the powder bed according to the designed part geometry until the powder reaches melting temperature and consequently achieves consolidation. When a new layer is to be scanned, a piston positioned under the substrate is moving downwards by the predefined layer thickness and a new layer of powder is deposited over the existing consolidated structure, as is illustrated in Figure 1. The above process is repeated until building of the entire part is completed.

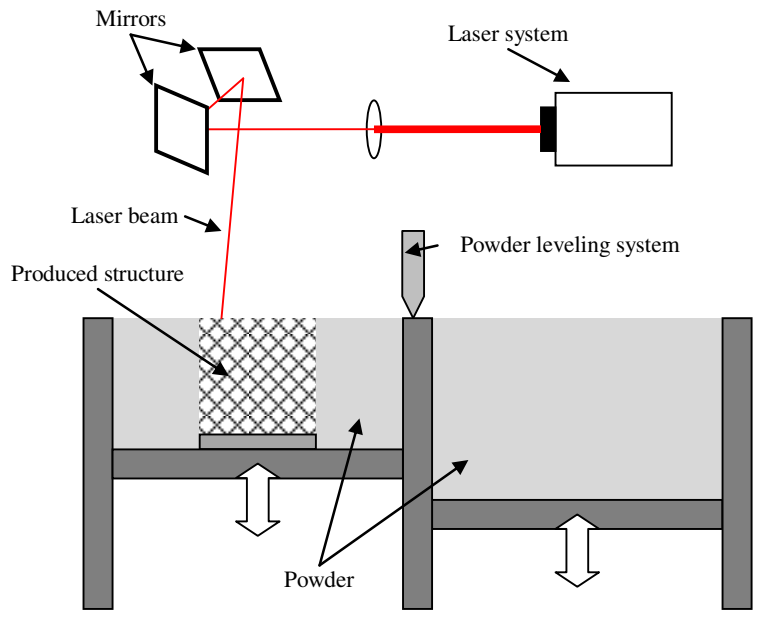

Fig. 1. Selective laser melting mechanism.

\subsection{The simulated lattice structure}

Lattice structure designs involve several variations of shape and size. The beams constituting the truss can be of various cross-sectional shapes, such as circular, square, rectangular, I-beam or hollow. In addition, the arrangement of the beams in space is matched to the type of application. The most widely used cellular topologies are the body centred cubic (BCC) and reinforced body centered cubic (RBCC), tetrahedral, pyramidal, 3D kagome, diamond textile, diamond and square collinear, octatruss and the wire-woven kagome. 
An example of the BCC lattice cell simulated part is depicted in Figure 2. The lattice unit cell is cubic, while its geometry is parametrically defined by its length 1 and strut diameter $\mathrm{D}$; typical values for length are $1-3 \mathrm{~mm}$, while strut diameter $\mathrm{D}$ ranges between $100-300 \mu \mathrm{m}$. The lattice unit cell size may vary in the $\mathrm{x}$, y directions, with typical values $2-$ $5 \mathrm{~mm}$. For a unit cell of $2.5 \mathrm{~mm}$ size, $2,5 \mathrm{~mm}$ length and $200 \mu \mathrm{m}$ nominal diameter, 50 layers are typically required in its fabrication, i.e. each layer has a thickness of $50 \mu \mathrm{m}$. The part is built by laser heating the four spots (one for each strut) in each layer.
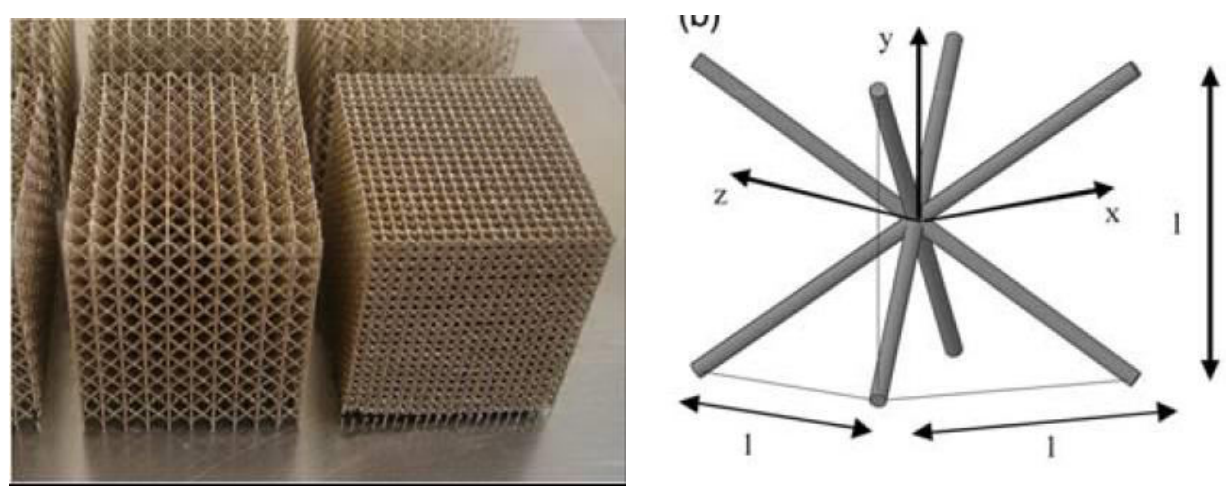

Fig. 2. The simulated BCC lattice cell geometry, lattice cores (left) and unit cell (right).

Different alloys of Al, Ti or Steel materials may be used in the manufacturing of the cellular structure. The presently developed SLM simulation methodology is demonstrated in the case of a cellular core made of Ti6Al4V material.

\section{Numerical Simulation Methodology}

\subsection{Thermal phenomena during the SLM process}

In SLM, heat transfer is occurring by all three mechanisms i.e. conduction, convection and radiation, as illustrated in Figure 3. Heat is provided to the part by the laser beam and then is transferred to the part volume via conduction through the solid and the powder material. Heat is lost from the system by means of convection and radiation of the free surfaces. Thus, the first law of thermodynamics for any material point in the part can be expressed as:

$$
Q_{L}=Q_{C D}+C_{C V}+Q_{R}
$$

where $Q_{L}, Q_{C D}, Q_{C V}, Q_{R}$ are the heat input from the beam, the conduction, the convection and the radiation losses respectively. Heat conduction for temperature-dependent conductivity can be expressed using the Fourier's law as:

$$
\frac{\partial}{\partial x}\left(k \frac{\partial T}{\partial x}\right)+\frac{\partial}{\partial y}\left(k \frac{\partial T}{\partial y}\right)+\frac{\partial}{\partial z}\left(k \frac{\partial T}{\partial z}\right)+\dot{q}=\rho C_{p} \frac{\partial T}{\partial t}
$$

where $k$ is the thermal conductivity, $T$ is the temperature of the part, $\dot{q}$ is the rate at which heat is supplied to the system, $\rho$ is the density of the material, $C_{p}$ is the specific heat capacity and $t$ is the interaction time between the beam and the material. 


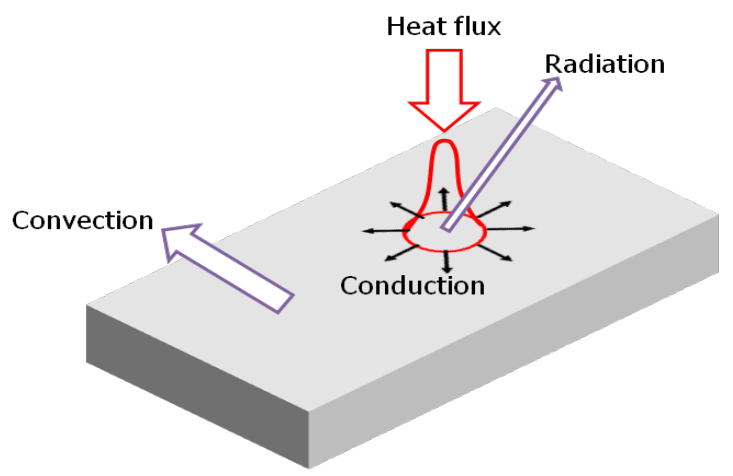

Fig. 3. Heat transfer mechanisms during Selective Laser Melting processing.

The initial conditions can be expressed as:

$$
T(x, y, z, 0)=T_{0}
$$

where $T_{0}$ is the ambient temperature, assuming that there is no pre-heating of the part.

In the presently simulated case, the heat of the laser beam is modelled as a heat flux. Radiation effects are omitted, since they are considered minor and heat losses only by convection are considered. Thus the boundary condition for the free surfaces of the part is:

$$
k \frac{\partial T}{\partial n}-\dot{q}_{s}+h\left(T-T_{0}\right)=0
$$

where $n$ is the vector normal to the surface, $\dot{q}_{s}$ is the rate of the heat input from the beam, $h$ is the heat transfer coefficient, $\sigma$ is the Stefan-Boltzmann constant and $\varepsilon$ is the emissivity. The above equation can be reduced, for the region heated by the beam, to:

$$
k \frac{\partial T}{\partial n}-\dot{q_{s}}=0
$$

as there is no convection. It is also reduced, for the rest of the free surfaces, to:

$$
k \frac{\partial T}{\partial n}+h\left(T-T_{0}\right)=0
$$

as there is no heat input by the beam.

\subsection{Thermal Material Properties}

For the Ti6Al4V material in solid form both the thermal conductivity and the enthalpy values are introduced in the present simulation as temperature-dependent properties, as presented in Figure 4. For the calculation of the thermal properties of the powder material it is assumed that the particles are spheres and there is no flattening of contact surfaces. Thus, the effective thermal conductivity of the powder bed is mainly influenced by the porosity of the powder bed $(\varphi)$, the diameter of the powder particles $\left(x_{r}\right)$ and the thermal conductivities of the fluid surrounding the powder particles $\left(k_{f}\right)$ and of the solid $\left(k_{s}\right)$ and is calculated using the following equation:

$$
\frac{k}{k_{f}}=(1-\sqrt{1-\varphi})\left(1+\frac{\varphi k_{r}}{k_{f}}\right)+\sqrt{1-\varphi}\left(\frac{2}{1-\frac{k_{f}}{k_{s}}}\left(\frac{1}{1-\frac{k_{f}}{k_{s}}} \ln \left(\frac{k_{s}}{k_{f}}\right)-1\right)+\frac{k_{r}}{k_{f}}\right)
$$


The density is considered to be constant with temperature but has different values for the powder $\left(2.64 \mathrm{~g} / \mathrm{cm}^{3}\right)$ and the solid material $\left(4.4 \mathrm{~g} / \mathrm{cm}^{3}\right)$. The substrate is made of steel and temperature-dependent thermal conductivity and enthalpy values have been introduced. All temperature depended material properties of titanium and steel alloys used in the present work have been obtained by [19] and [20].

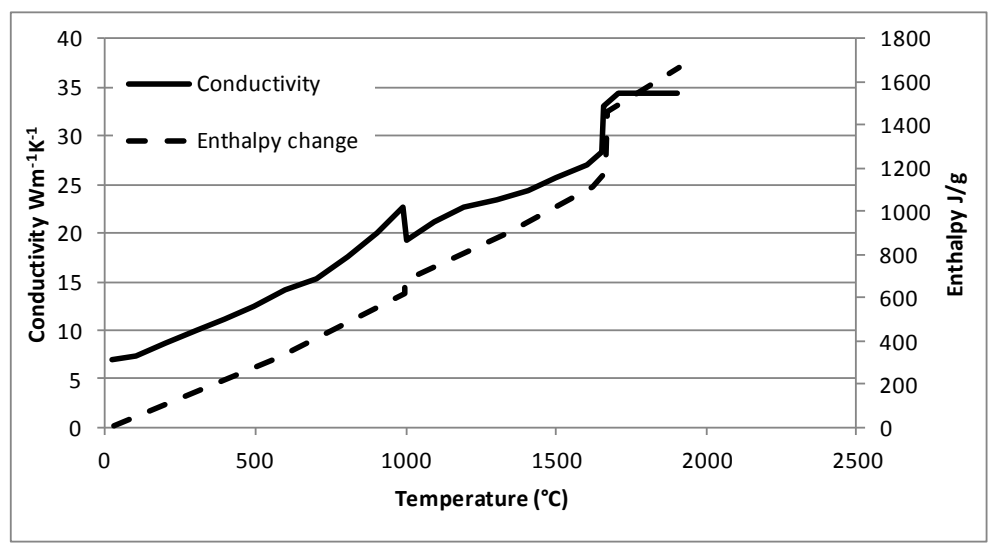

Fig. 4. Temperature-dependent Ti6Al4V thermal conductivity and enthalpy change [19].

\subsection{Numerical Model Development}

A finite element model has been developed in Ansys FE code for the thermal simulation of an open lattice core structure manufacturing using the SLM process. During the process the laser beam interacts with the top layer of the material for a period of time called irradiation time. After the beam has irradiated a spot, it moves to the next spot with speed equal to the scanning speed. Once a layer has been created, powder is deposited on top of it and the next layer creation initiates. The time interval between two successive layers creation is called recoating time. The nominal laser process parameters used in the present simulation are: spot diameter $100 \mu \mathrm{m}$, heat flux coefficient 0.78 , irradiation time $1000 \mu \mathrm{s}$, scanning speed 1 $\mathrm{mm} / \mathrm{s}$, laser power $200 \mathrm{~W}$ and exposure times of $500 \mathrm{~ms}, 750 \mathrm{~ms}$ and $1000 \mathrm{~ms}$.

The parametric FE model developed represents one unit cell structure, plus a substrate layer. The model is meshed using 8-nodes hexahedral SOLID70 elements. Each node has a single degree of freedom (DOF), temperature. Different mesh densities have been trailed for process simulation. In the different FE models, the defined element size has been correlated to the diameter of the laser beam. Thus, initially, a model with 2 elements / laser diameter has been developed resulting to a total of 145,656 nodes and 137,500 elements. A second, denser FE model, with 4 elements / laser diameter has been built leading to 1,081,306 nodes and 1,050,000 elements. Finally, the largest FE model built has 8 elements / laser diameter, 8,522,606 nodes and 8,400,400 elements. The different models having increasing mesh density are presented in Figure 5. From the above FE models the second one has been used for the calculations that are presented hereunder, as the first one was not sufficiently dense to provide a good prediction of the final strut geometry, while the last leaded to extremely long computational times, without adding significant improvements in the achieved results. Therefore, a mesh of 4 elements per strut cross section diameter can be considered converged for the current simulation. 

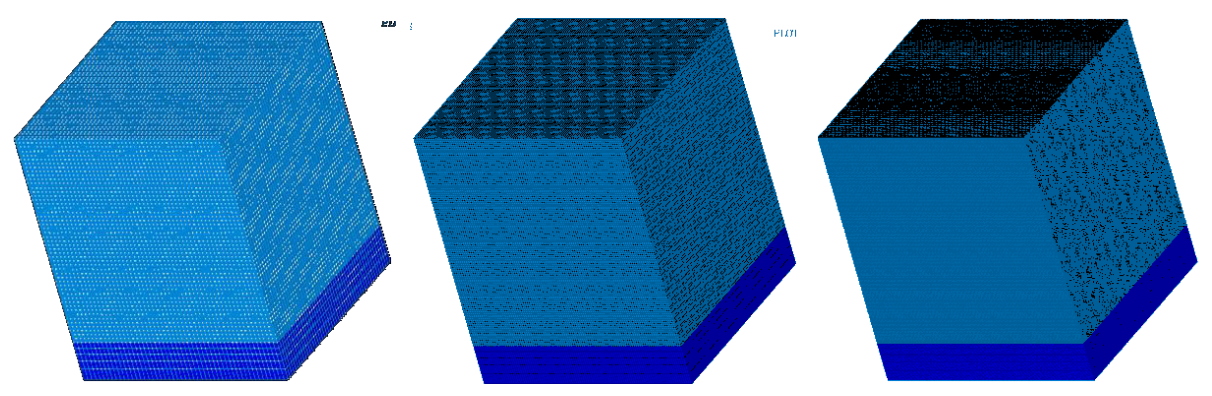

Fig. 5. FE models developed with different mesh densities (representing a unit cell of $2.5 \mathrm{~mm}$ ).

Initially, all the elements of the FE model (shown in Figure 4) are given material properties corresponding to the powder form of the material. During laser scanning, the material properties of elements under the spot which reach melting point are changed to the properties of solidified material. A technique known as 'element birth and death' has been used to simulate the gradual build-up of the part. At the initiation of the manufacturing process all elements are in the 'dead' status, which means that there are present in the model, but they do not contribute to the stiffness matrix, since their properties are divided by a very high property reduction factor. As the building process is evolving, the elements of every new powder layer that is added on the table are changed to 'alive' status, meaning that they are given their actual material properties. Using the specific modelling technique the necessary for creating an updated FE model and transfer to it all previous results each time a new layer of material is added, is eliminated. Even if this approach leads to largeer FE models in the first steps of the simulation, this disadvantage is compensated by the convenience in programming and the ensurance of correct transfer of temperatures between subsequent steps.

It has to be mentioned that generally the simulation of the SLM process is related to high computational costs. This is mainly due to the significant difference of the scales of the final product and the area where thermo-mechanical processes take place; i.e. the area around the laser-irritated spot, leading to the necessity of very dense FE models. Even for the case of the simulation of a single unit-cell $(2.5 \mathrm{~mm} \times 2.5 \mathrm{~mm} \times 2.5 \mathrm{~mm})$ the layer thickness is $50 \mu \mathrm{m}$ and the laser beam diameter is $100 \mu \mathrm{m}$.

The heat input in the material by the laser beam is modelled as a constant heat flux applied on the elements that their whole face is covered under the laser spot. An example of this approach is shown in Figure 6, where the perimeter of the laser spot is marked with red colour and the elements that the heat flux is applied are hatched, i.e. the laser spot is represented by 12 elements. The magnitude of the heat flux is derived by the following equation:

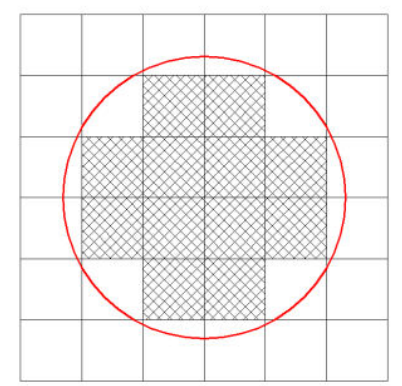

Fig. 6. Elements on which heat flux is introduced. 


$$
\dot{q}_{s}=\frac{a P}{n b^{2}}
$$

where $\mathrm{P}$ is the laser power, a is the material's absorptivity and $\mathrm{n}$ is the number of elements "laying" inside the laser spot and $b$ is the size of the elements edge. In the present cases the value of 0.3 for the absorption coefficient a is taken following the analysis of Roberts [19].

\subsection{Simulation results of BCC unit cell structure}

An implicit time-integration scheme was used for the transient solution of the thermal problem during SLM process application. The Ansys sparse direct equation solver was used for obtaining the solution, while the full transient and Newton-Raphson options were selected. Typical solution times using an i7 personal computer have been of the order of three to five days. The temperature field evolution during the SLM process has been calculated. During processing of a layer, the distribution of temperature the 'layer below' (solidified in the previous layer step) gives indication about material re-melting, which is useful in the prediction of the fusion volume and provides an indication about the quality of material continuity. In Figure 7(left) the temperature field on a produced strut (on the top layer and on the 'layer below') is presented, showing the elements that have reached material's melting temperature. The BCC cell final shape has been determined by the elements that exceeded melting temperature during the entire process evolution. An indicative predicted BCC unit cell shape is presented in Figure 7(right), comprising the FE model element that have reach the material melting temperature at any stage of the process.

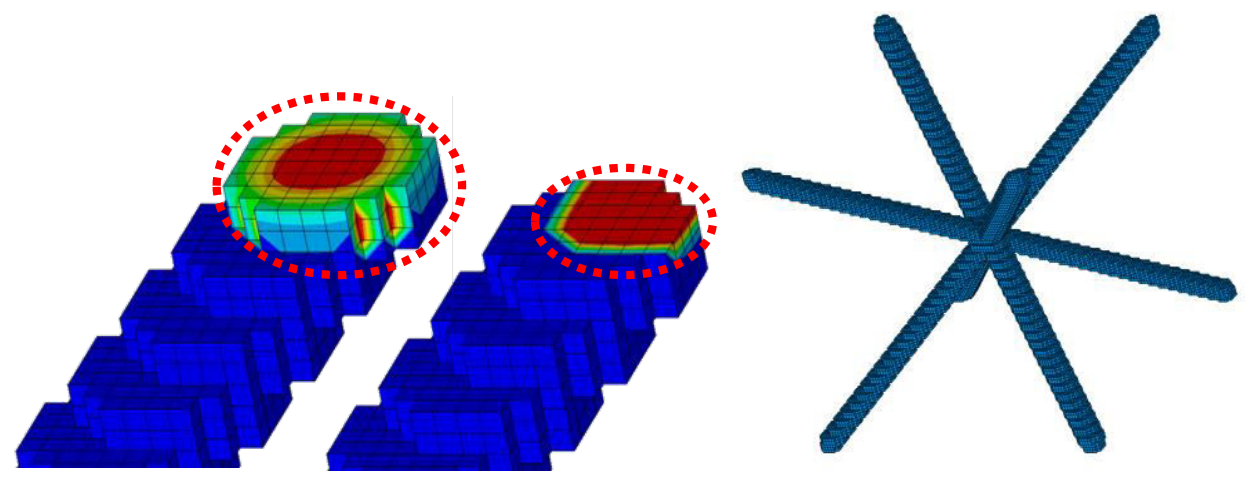

Fig. 7. Temperature field developed on a build strut (left) and predicted final BCC structure shape (right).

The simulation results have been validated with respective experimental measurements presented in [5]. To this scope the BCC structure strands diameters, as predicted by the numerical simulations and as measured at a SLM created structure, are compared. As strands cross-section is neither perfectly circular, nor perfectly constant along their length, strands diameter has been estimated at different positions. The estimated strand diameters are presented in the diagram of Figure 8, where the numerical simulation results concern laser beam energies of $0.1 \mathrm{~J}, 015 \mathrm{~J}$ and $0.2 \mathrm{~J}$ (corresponding to laser power of $200 \mathrm{~W}$ and exposure times of $500 \mathrm{~ms}, 750 \mathrm{~ms}$ and $1000 \mathrm{~ms}$ ). From this diagram it can be observed that the comparison of numerical results to experimental measurements show similar trends and have similar standard deviations. 


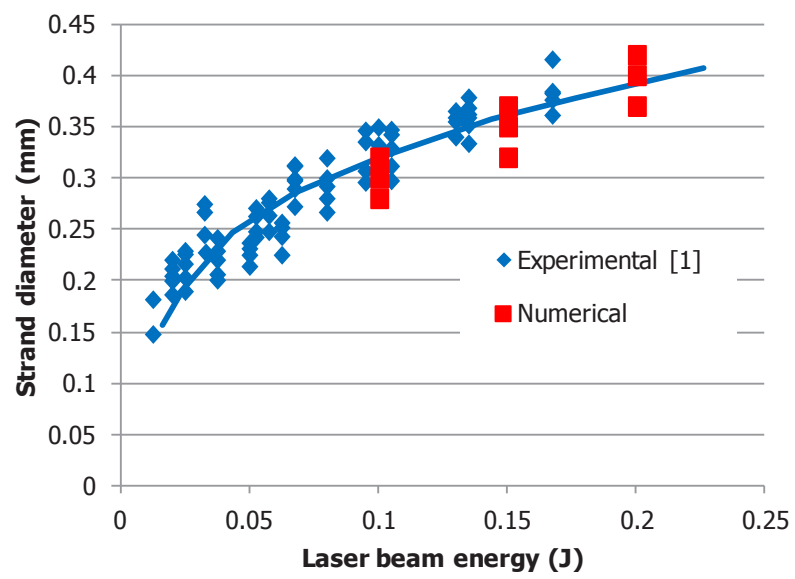

Fig. 8. Comparison of calculated strand diameter values to experimental measurements for different laser beam energy levels.

Consequently the predicted unit-cell geometrical characteristics (strand length and diameter) can be used in combination with an analytical of numerical methodology in order to predict the resulting material apparent mechanical properties. Such methodologies have been developed by the authors and have been presented in previous works (see [14] and [21]). Thus, stress-strain curves as function of geometrical characteristic prediction by the present simulation, as presented in Figure 9, can be predicted.

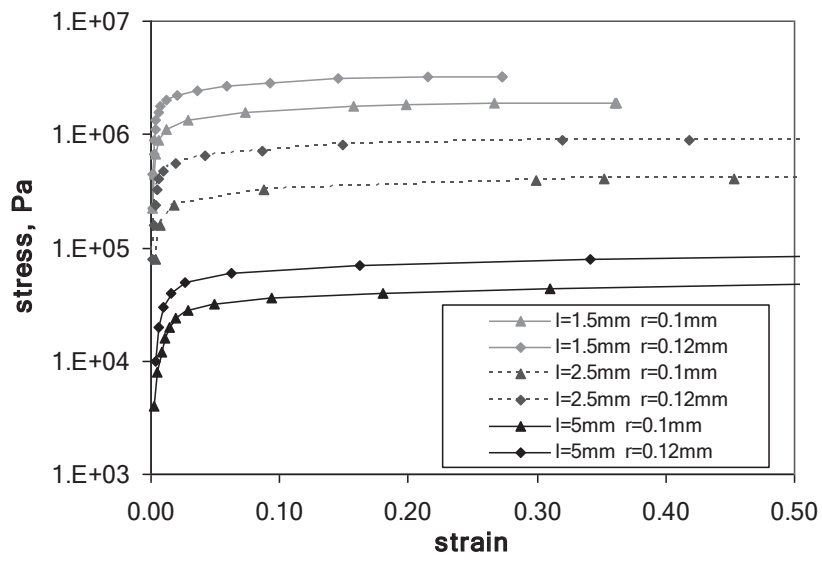

Fig. 9. Influence of unit-cell size and strut radius on the response of the bcc-type core [21].

\section{Conclusions}

An efficient simulation of SLM manufacturing process of open-lattice cellular structures is a very challenging task, which however is in general possible, at least at small scales, as presently demonstrated for the case of a BCC unit cell structure. The numerical simulation can be used to predict the final geometry of the open-lattice material and the temperature history, as function of AM process parameters applied. Thus, the present numerical simulation approach can be a useful tool for the first-time right selection of SLM process parameters which result to an open-lattice structure with desired material properties. 


\section{References}

1. E. Ptochos, G. Labeas, J. Sand. Struct. Mater. 14 (2012)

2. R. Gümrük, R. Mines, Int. J. Mech. Sc. 68 (2013)

3. G. Labeas, M. Sunaric, V. Ptochos, Engineering Against Fracture, S. Pantelakis and C. Rodopoulos (Springer, Netherlands, 2009)

4. Y. Shen, S. Mckown, S. Tsopanos, C. Sutcliffe, R. Mines, W. Cantwell, J. Sandw. Struct. Mater. 12 (2009)

5. R. Mines, S. Tsopanos, Y. Shen, R. Hasan, S. McKown, Int. J. Impact Eng. 60 (2013)

6. J. Liu, F. Lu, D. Fang, H. Zhao, Int. J. Protect. Struct. 2 (2011)

7. G. Labeas, E. Ptochos, Plast. Rubber Comp. 42 (2013)

8. M.G. Rashed, M, Ashraf, R.A.W. Mines, P. J. Hazell, Mater. \& Design 95 (2016)

9. C. Yan, L. Hao, A. Hussein, S. Bubb, P. Young, D. Raymont, J. Mater. Proc. Techn. 214 (2014)

10. I. Gibson, D. Rosen, B. Stucker, Additive manufacturing technologies (Springer, New York, 2010).

11. J. Kruth, M. Leu, T. Nakagawa, CIRP Annals - Manuf. Techn. 47 (1998)

12. S. Mellor, L. Hao, D. Zhang, Int. J. Prod. Econ. 149 (2014)

13. S. Park, D. Rosen, S. Choi, D. Duty, Addit. Manufact. 1-4 (2014)

14. C. Yan, L. Hao, A. Hussein, D. Raymont, Int. J. Mach. Tools Manuf. 62 (2012)

15. A. Hussein, L. Hao, C. Yan, R. Everson, Mater. Des. 52 (2013)

16. A. Foroozmehr, M. Badrossamay, F. Foroozmehr, S. Golabi, Mater. Des. 89 (2016)

17. N. E. Hodge, R. M. Ferencz and J. M. Solberg, Comput. Mech. 54 (2014)

18. J.-R. Zhuang, Y.-T. Lee, W.-H. Hsieh, A.-S. Yang, Optics \& Laser Techn. 103 (2018)

19. I. A. Roberts, Investigation of residual stresses in the laser melting of metal powders in additive layer manufacturing, Thesis submitted in partial fulfilment of the requirements of the University of Wolverhampton for the degree of Doctor of Philosophy (2012)

20. K. C. Mills, Recommended Values of Thermophysical Properties for Selected Commercial Alloys, (Woodhead Publishing, Cambridge, 2002)

21. G. N. Labeas and M. M. Sunaric, Strain 46 (2010) 